C. I. F., XIX-XX (1993-1994) 27-38

\title{
FUNCIONAMIENTO ENUNCIATIVO DE LA DETERMINACIÓN PARTITIVA EN FRANCÉS*
}

\author{
Leyre Ruiz de Zarobe** \\ Universidad del País Vasco
}

\begin{abstract}
RESUMEN. La determinación partitiva del francés se ha asociado tradicionalmente con el universo semántico de los nombres incontables, y con la actualización de un valor de cuantificación. En este artículo intentamos mostrar que esa asociación es insuficiente para dar cuenta del funcionamiento discursivo de la determinación partitiva, adoptando una nueva categorización nominal y analizando las operaciones enunciativas subyacentes a la determinación partitiva.

RÉSUME. La déterminacion partitive du français a été associée traditionnellement a l'univers sémantique des noms indénombrables et à l'actualisaton d'une valeur de quantification. Dans cet article on essaie de montrer que cette association est insuffisante pour rendre comnpte du fonctionnement discursif de la détermination partitive, adoptant une nouvelle catégorisation nominale et analysant les opérations énonciatives sous-jacentes à la détermination partitive.
\end{abstract}

Los distintos gramáticos asocian a la determinación partitiva, para hacerse actualizable, una sub-clase de nombres, que ha solido denominarse "masiva". Esto presupone, empleando una terminología más corrientemente manipulada, una distinción previa entre la clase de nombres "indénombrables" o no contables, opuesta a la clase de los "dénombrables" o contables. Así, se dice que el partitivo se emplea, de manera general y esencial, con los nombres "indénombrables" para indicar que no se considera más que una parte del todo designado por el nombre. En otros términos, los dos elementos esenciales de la definición semántica sobre el partitivo son:

- por una parte, la asociación del partitivo al universo de lo no enumerable

- por otra parte, la asociación del partitivo al universo de la cuantificación

* Recibido el 9 de febrero de 1993.

** Doctora en Filología Francesa y Profesora Titular de Lingüística Francesa en la Universidad del País Vasco, Vitoria-Gasteiz. C/. Marqués de Urquijo, s/n. - 01006 Vitoria (Alava). 
Vamos a intentar mostrar que esta definición no resulta suficiente para dar cuenta del funcionamiento discursivo del partitivo. En primer lugar, nos parece necesario pensar de nuevo los hechos en función de una nueva categorización del sustantivo, tomada de Culioli. Culioli ${ }^{1}$ distingue las categorías nominales siguientes: 1) discreto 2) denso 3) compacto. A partir de esta distinción, vamos a intentar analizar las operaciones específicas subyacentes a la determinación partitiva, mostrando cómo el determinante partitivo es marca en superficie de un cierto número de operaciones subyacentes en la lengua (Culioli).

A este respecto, y más globalmente, señalamos que nuestro marco teórico general previo se sitúa en las teorías lingüísticas de la enunciación, particularmente la teoría desarrollada por Culioli².

Culioli habla de la categoría /discreto/ "lorsqu' on a le caractère individuable et tout ce qui en découle" 3 ; otra propiedad es la posibilidad de obtener una clasificación ordinal: $1^{\mathrm{a}}, 2^{\mathrm{a}}, 3^{\mathrm{a}}$ ocurrencia. Ejemplos de /discreto/: "livre, chat, enfant". Habla de la categoría /compacto/, cuando "on a affaire à l'insécable, à du prédicatif nominalisé, sur lequel on ne peut effectuer aucun prélèvement" 4 . Ejemplos de /compacto/: "courage, difficulté, plaisir". Finalmente, habla de la categoría /denso/, cuando

il s'agit de notions qui renvoient à une réalité avec laquelle vous pouvez effectuer des opérations éventuellement d'ordre discret, mais par l'intermédiaire à ce moment-là d'un dénombreur. [...] Prenons un exemple: "l'eau". Ou bien vous dites "un verre d'eau", dans ce cas vous avez un dénombreur [...]; ou bien vous dites: "de l'eau", "un peu d'eau" et sauf si vous avez un étalon, quel qu'il soit, vous n'avez pas la possibilité de dire: "une fois de l'eau, deux fois de l'eau", "une fois un peu d'eau", "deux fois un peu d'eau"s.

Ejemplos de /denso/: "farine, lait, viande".

Hay que decir que las propiedades /discreto/, /denso/, /compacto/ no son propiedades físicas, sino propiedades lingüísticas. No se trata tampoco de propiedades de las

1. A. Culioli. Transcription du séminaire de DEA. Recherche en linguistique. Université de Paris VII, 1983.

2. La teoría de Culioli ha sido transmitida de manera bastante dispersa. Puede seguirse una visión de conjunto en Transcription du séminaire de DEA. Recherche en Linguistique. Université de Paris VII, 1983; o en la recopilación reciente Pour une linguistique de l'énonciation. Opérations et représentations. (tome 1 et 2), Ophrys, 1990.

Muy esquemática y reductoramente destacamos que la actividad lingüística se concibe como la puesta en relación de tres niveles: el nivel de las representaciones mentales; el nivel de la actividad del lenguaje, donde se inscriben las marcas de esas operaciones mentales; y el nivel de las representaciones metalingüísticas, donde se elabora el sistema de operaciones que tipifica las propiedades formales de la interlocución.

3. A. Culioli, Transcription... op. cit.

4. A. Culioli, "A propos de 'quelque"', en S. Fisher et J.J. Franckel (eds), Linguistique, énonciation: aspects et détermination. París: EHESS, 1983, p. 26.

5. A. Culioli, Transcription... op cit., p. 103. 
unidades léxicas, ya que algunas operaciones de determinación modifican esas propiedades, como veremos 6 .

\section{El /denso/}

Vamos a iniciar el análisis con la categoría nominal /denso/, ya que, aunque bajo otras denominaciones que presuponen categorizaciones nominales diferentes, se trata de la categoría que se asocia en primer lugar al partitivo.

Así, en:

(1) -Qu'est-ce qu'il boit? -Du vin

la determinación partitiva se opera sobre el objeto masivo "vin". En un primer enfoque, y retomando la definición de Martin:

Un objet massif est tel que ses parties ne sont pas de nature différente de la nature du tout?

Es el "critère de sous-ensemble" (una parte del vino sigue siendo vino), al que hay que sumar el "critère de réunion" (vino añadido a vino resulta vino).

En este caso "le vin" remite a la totalidad de la especie sustancial, es decir, su referencia es genérica. Sobre esta masa "vin", se extrae una cierta cantidad, una cantidad indefinida, indeterminada, imprecisa, de vino. El partitivo efectúa una operación de "prélèvement" de una cantidad. Se opera un corte, una delimitación de la noción, lo que corresponde al "prelèvement", en donde se aísla una parte.

Esta operación sobre la propiedad/denso/ es una operación efectuada sobre la "quotité", es decir, sobre la cantidad no definida, concebida globalmente, al margen de la cardinalidad.

(2) Il boit du vin qui est à table

Esta ocurrencia del partitivo es de una frecuencia mucho menor que la ocurrencia contemplada más arriba. El uso del partitivo aquí se relaciona no con una clase genéri$\mathrm{ca}$, sino con un objeto preciso e identificable. "Le vin" posee en este caso una referencia determinada. Sobre este objeto "vin", definido, se efectúa la operación de "prélèvement", y se obtiene como resultado una noción específica.

Si aquí el partitivo es conmutable por el conjunto "preposición + "ce"”: "Il boit de ce vin qui est à table", lo que confirma, según Kupferman", la referencia definida del artículo, algunos verbos excluyen esta posibilidad referencial. A este respecto, el autor

6. A.M. Leonard, "Etude différentielle de quelques indéfinis anglais", en S. Fischer et J.J. Franckel (éds), Linguistique, énonciation: aspects et détermination. París: EHESS, 1983, p. 48.

7. R. Martin, "De la double extensité du partitif", Langue Française, 57 (1983) p. 36.

8. L. Kupferman, "L'article partitif existe-t-11?", Le Français Moderne, 47 (1979) pp. 1-16. 
cita los verbos "voir, montrer, apporter", como excluyentes de la ocurrencia del partitivo con interpretación específica:

*Je n'ai pas vu du gâteau de Marie

es agramatical, frente a

Je n'ai pas mangé du gâteau de Marie.

(3) Ceci est du vin

En este enunciado, un objeto bien determinado ("ceci") es asimilado al objeto "le vin". La operación efectuada puede denominarse de atribución.

Esta operación permite usos del partitivo en los que se resalta el valor cualitativo de la noción:

(4) Ça, c'est du vin!

en el sentido de que ese vino reúne las mejores cualidades que puede tener un vino.

El $\mathrm{N}$ afectado por el partitivo en los casos contemplados hasta aquí se caracteriza por poseer un tipo de referencia: la referencia de los nombres de masa. Esta ha sido definida por medio del axioma de la referencia homogénea:

Toutes les parties d'une quantité de $\mathrm{x}$, qui sont elles-mêmes des quantités de $\mathrm{x}$, peuvent devenir les parties d'une autre quantité de $\mathrm{x}^{9}$.

En este sentido, pero bajo otra perspectiva, se puede decir que la extensión de un $\mathrm{N}$ masivo como "vin" está constituido por el conjunto de ocurrencias no distinguibles entre sí que son vino, y por tanto aquélla se percibe como homogénea.

Dentro de la categoría /denso/ asistimos a un fenómeno que hay que considerar igualmente. Es posible operar el "prélèvement" de una cantidad de elementos contables sobre una noción considerada /densa/. Así encontramos normalmente enunciados como:

(5) Il préfère une viande très rouge

(6) J'ai acheté des vins excellents

(7) Donnez-nous deux cafés (deux tasses de café)

De /denso/ hemos pasado a la categoría de /discreto/. Esta operación ha sido posible por medio de un "dénombreur", expreso en (5) y (6) y no expreso, pero expresable, en (7).

Martinet que rechaza la oposición en francés "dénombrable/indénombrable", afirma que la elección del partitivo no está condicionada por el valor del núcleo, sino textualmente:

par le désir d'exprimer qu'il y a une quantité non comptée d'un certain type de choses. Tout nom français peut donc apparaître avec ou sans le partitif ${ }^{10}$.

9. M. Galmiche, "Note sur les noms de masse et le partitif", Langue Française, 72 (1986) p. 46; citando a A. ter Meulen. Substances, Quantities and Individuals, Bloomington, Diss. Stanford Univ., IULC, 1980.

10. A. Martinet. Grammaire fonctionnelle du français. París: Didier, 1979, p. 45. 
Esta última afirmación no parece corresponderse enteramente con los hechos del uso del partitivo, como ha mostrado Spence ${ }^{11}$ en un interesante artículo en el que da cuenta del paradigma de la ocurrencia del partitivo según clases semánticas.

Tal visión de los hechos contiene gérmenes de elementos que apuntan a una explicación del fenómeno. Damourette-Pichon presentaban el fenómeno así:

En français moderne toute substance peut être envisagée d'un point de vue ou de l'autre. C'est-à-dire que, par le répartitoire de putation, elle peut être estimée, dans l'ordre de la quantitude, suivant deux systèmes différents: ou bien l'on donne une indication sur la grandeur globale de la masse de substance que l'on prélève sur la totalité de la substance de l'espèce donnée (putation massive); ou bien l'on fournit un renseignement sur le nombre des individus que l'on prélève dans l'ensemble de cette espèce, conçue comme décomposable en unités particulières (putation numérative) ${ }^{12}$.

Así pues, según este punto de vista, no existirían sustancias masivas y enumerativas "in esse", sino dos maneras distintas de concebir la sustancia: de manera global (es el caso del masivo) o de manera individualizadora (es el caso del numerativo).

Wilmet afirma a este respecto:

En accord ou non avec la perception que nous avons d'un objet du monde 1) discret ou 2) dense, les substantifs sont passibles d'une représentation 3) numérative ou 4) massive ${ }^{13}$.

En una perspectiva diferente, Galmiche resume su posición así:

Tout nom comptable peut avoir un emploi massif et (inversement) tout nom massif peut avoir un emploi comptable ${ }^{14}$.

defendiendo con ello un cambio de empleo del nombre.

En cualquier caso, dado que la marca lingüística nominal no cambia en el caso del masivo o del numerativo, nos parece legítimo pensar que la masividad o la enumeración es factor de la marca determinante.

11. N. Spence, "Partitive and Mass-Nouns in French", Romanische Forschungen, 95 (1983) pp. 1-22.

12. J. Damourette et E. Pichon, Des mots à la pensée. Essai de grammaire de la langue française. 1911-1927 (7 vol.). París: D'Artrey, 1987, p. 425.

13. M. Wilmet, La détermination nominale. Quantification et caractérisation. París: Presses Universitaires de France, 1986, p. 50.

14. M. Galmiche, "A propos de la distinction massif/comptable", Modèles Linguistiques, 1988. 


\section{EI /Discreto/.}

El partitivo, en esencia, no está considerado en asociación con la categoría /discreto/, ya que ésta pertenece al universo de lo individualizable y de lo enumerable.

Sin embargo, encontramos naturalmente en francés enunciados como:

(8) J'ai mangé du veau à midi

"Veau" se caracteriza por la propiedad inherente de la contabilidad, pero aquí comprendemos que no se trata de un nombre contable, sino de un masivo, en un uso en el que el determinante precisamente ha contribuido al establecimiento del rasgo masivo.

$\mathrm{Al}$ enunciar "J'ai mangé du veau à midi", partimos no de una masa, sino de una entidad individualizada, y por lo tanto organizada en partes. En este caso, la parte extraída no es una parte cualquiera e indeterminada, sino una parte específica y determinada, que corresponde a la parte útil para la actividad humana: la carne ${ }^{15}$.

Sin embargo, el establecimiento de la significación de los enunciados de este tipo está en estrecha relación con las condiciones enunciativas. Así, la enunciación de "du veau" en un contexto como el del zapatero remitirá a una parte distinta de la carne, al cuero. Asimismo, el locutor, en un contex to concreto, designará, al enunciar "du chien", la piel de éste, y su carne en la expresión "dur comme du chien". En consecuencia, la significación de estos enunciados y por tanto la selección de la parte que ha de extraerse no está determinada por la inherencia partitiva inequívoca del objeto -es el caso de los nombres de masa-, sino que su establecimiento es función de las condiciones enunciativas compartidas por el locutor e interlocutor.

Vamos a tocar ahora un punto general en relación con la determinación partitiva. La repartición de la masividad o de la contabilidad se acomoda en francés a las flexiones de número del sustantivo ${ }^{16}$. La masividad se acomoda generalmente al singular, traduciendo la continuidad. Ya que concebir una sustancia masivamente es abstraer toda delimitación, considerarla únicamente como continua. Por otra parte, la contabilidad se acomoda al plural, traduciendo la discontinuidad. Concebir una sustancia numerativamente es hacerla divisible, descomponerla en unidades, y concebirla así pues de manera discontinua. Así pues, como esquematizan Damourette-Pichon, el reparto continuo/discontinuo se sobreimprime a la masividad/contabilidad del sustantivo, permitiendo un cruce de los dos bloques para obtener cuatro resultados diferentes que no vamos a analizar aquí.

Hemos creído necesario tocar este punto general para introducir un tipo ocurrencial del partitivo dentro de esta categoría, que podría considerarse como una manifestación de la visión de continuidad en concurrencia con la visión de discontinuidad. Dicho de otra manera, en ciertas condiciones enunciativas, el enunciador opta por una 
concepción masiva en lugar de una concepción contable. Los enunciados siguientes son ilustraciones de este mecanismo:

(9) Il y a du champignon dans cette forêt (Kleiber, 1989)

(10) Ce libraire est très spécialisé; il ne vend que du livre d'art et du roman policier (Galmiche, 1988)

frente a :

(11) Il y a des champignons dans cette forêt

(12) Ce libraire est très spécialisé; il ne vend que des livres d'art et des romans policiers.

Si como afirmaba Martinet todo $\mathrm{N}$ en francés es susceptible de asumir un determinante masivo así como un determinante contable, el enunciador, en el primer bloque de enunciados elige preferentemente la concepción masiva del $\mathrm{N}$ en lugar de su concepción enumerativa o contable. Si el segundo bloque no presenta problemas de interpretación, pues un $\mathrm{N}$ discreto y por lo tanto contable se presenta bajo una concepción que traduce la discontinuidad, habrá sin embargo que justificar la elección de la presentación de ese $\mathrm{N}$ bajo una concepción masiva.

Si seguimos la interpretación de Kleiber ${ }^{17}$, al enunciar "Il y a des champignons dans cette forêt", individualizamos la noción "champignon", suponemos un conjunto de unidades distintas, remitimos a la tipología diversa de los champiñones, a los "occurrences constitutives"18 de la noción. "Champignon" tiene aquí una referencia individualizada.

Sin embargo, una enunciación como "Il y a du champignon dans cette forêt" presenta el referente de manera homogénea, y por consiguiente el enunciador prima no las ocurrencias en tanto que distintas, sino la identidad entre las ocurrencias. Este último enunciado puede ser propio de un micólogo, por ejemplo, que quiere poner en relieve el hecho de encontrar la especie "champignon" en un bosque, sin tomar en consideración si se trata de grandes, pequeños, comestibles, venenosos etc. Así pues, sería esa "présentation homogène des occurrences constitutives du référent" 19 la que justifica la elección del partitivo masivo para un $\mathrm{N}$ contable. En realidad, el SN tiene un efecto de sentido pluralizante, ya que se entiende que lo que hay en el bosque son "des champignons"; ello es fruto de la esencia contable de "champignon". "Du" actualiza una operación de masificación del $\mathrm{N}$, entendiendo por ésta la homogeneización de los elementos que constituyen el referente.

Estas ocurrencias del partitivo corresponden a una creación libre por parte del enunciador, que podrá fabricar así enunciados como:

(13) Il y a de la voiture sur la route

(14) On va casser du flic

17. G. Kleiber, “'Le’ générique: un massif?”, Langages, 94 (1989) pp. 73-109.

18. G. Kleiber, op. cit.

19. G. Kleiber, op. cit., p. 91. 
En uno y otro caso, el referente de $\mathrm{N}$ se presenta homogeneizado.

Esta ocurrencia del partitivo como presentación de la "masificación" con sentido pluralizante es señalada por Galmiche ${ }^{20}$ como un argumento para defender el parentesco del determinante partitivo con el artículo indefinido "des", más que con el artículo indefinido "un", habiendo sido esto último lo más habitual en los estudios que intentaban mostrar la similitud del partitivo con el indefinido. Así pues, y retomando únicamente el argumento que nos interesa aquí, considera la posibilidad de tener, por una parte, términos que aparecen sistemáticamente en plural, y por otra parte, términos de masa, parasinónimos de esos términos en plural, con referencia pluralizante. El hecho curioso es que esos términos en plural difícilmente se ven afectados por una operación de "dénombrement", lo cual justifica seguramente su asimilación a la forma masiva. Ej:

(15) acheter de la pharmacie des médicaments

vendre de l'armement des armes (Galmiche, 1986)

En estos casos vemos que un término de masa y un término contable tienen el mismo tipo de referencia.

Existe un uso del partitivo diferente a los usos que hemos visto hasta aquí. Veamos:

(16) Dans tout ancien professeur de philosophie, il y a de l'apôtre (P. Bourget) ${ }^{21}$.

En este enunciado, significamos "dans tout ancien professeur de philosophie il y a quelque chose de la qualité apôtre", ya que se concibe alguna participación en el conjunto de las calidades/cualidades que caracterizan a un "apôtre". Sobre la noción /être apôtre/, el partitivo va a llevar a cabo una retención de ciertas propiedades cualitativas características de "apôtre". Esto significa que la ocurrencia del partitivo produce una lectura cualitativa selectiva de "apôtre".

Este funcionamiento se encuentra asimismo afectando a los nombres propios:

(17) Il y a du Don Juan en lui significando "Il y a en lui quelque chose qui tient du caractère de Don Juan". El determinante permite participar al actante de la noción "Don Juan", en tanto que propiedad.

Encontramos asimismo en esta categoría /discreto/ al partitivo como marca de operación atributiva, por medio de la cual se acentúa la dimensión cualitativa de la noción :

(18) Ça, c'est de la voiture!

Este uso constituye

une des sources les plus favorables pour le passage du discret au continu ${ }^{22}$.

20. M. Galmiche, "Note sur les noms...". Op. cit.

21. Ejemplo citado por M. Grevisse.

22. R. Martin, op. cit. p. 40. 
Esta operación también puede hallarse afectando a los nombres propios:

(19) C' est du vrai Zola

\section{El /Compacto/.}

Vamos a analizar finalmente el funcionamiento enunciativo del partitivo con la categoría del /compacto/. Como vimos, en este caso tratamos con "l'insécable" en sentido estricto, sobre el que no se puede efectuar ningún "prélèvement". Esto significa que no se puede llevar a cabo operaciones que afectan a la cantidad o a la calidad, ya que el compacto no autoriza ninguna partición. Así, en el enunciado:

(20) Cette femme a du mérite

"du" no es la marca de una partición. "Du" significa "peu importe quantitativement combien ou qualitativement iequel", ou "je suis incapabie de dire quantitativement combien ou qualitativement lequel", en todo caso, una ocurrencia no nula. "Du" marca aquí la existencia de un valor no nulo.

Este empleo del partitivo era concebido por Guillaume de una manera que no nos parece muy diferente. Guillaume, distinguiendo dos tipos de posiciones de pensamiento:

La position de pensée dans l'inactuel donne des images infinies, la position de pensée dans l'actuel, des images finies aux contours flottants (art. du, de la) ou arrêtés (art. un).

L'image est infinie lorsque l'idée n'est adéquate qu'à elle-même; elle est finie lorsque l'idée est adéquate a quelque réalité sensible ${ }^{23}$.

daba la explicación siguiente :

"Le malheur" est une idée infiniment étendue dans l'abstrait. [...] ; “du malheur", une idée réduite aux proportions d'un effet sensible [...]; " "un malheur", la même idée réduite aux dimensions d'un fait déterminé.

"La bonté" est l'idée d'une qualité morale, sans plus [...]; "de la bonté" sous-entend un cas particulier de possession ou d'emploi de cette qualité" 24.

Culioli25 afirma que la categoría del /compacto/ implica una calificación subjetiva, lo que permite hablar de intensidad, de calidad, de una sensación o sentimiento. Así tendremos:

23. G. Guillaume, Le problème de l'article et sa solution dans la langue française. París-Québec: Nizet-P. Univ. Laval, 1975, p. 104.

24. G. Guillaume, op. cit., pp. 105-106.

25. A. Culioli, "A propos de "quelque'”... op. cit., p. 26. 
(21) Ayez de la patience avec lui!

(22) Elle montre du courage dans cette affaire

Es posible modular la categoría de lo compacto desde el punto de vista de la intensidad del nombre y no de su extensión. Ello nos permite crear, por ejemplo: "une grande patience". Esta operación no es posible para la categoría /denso/

La operación señalada para el compacto se encuentra asimismo en los enunciados del tipo:

(23) Il fait de la bronchite/de la fièvre/de l'hypertension al lado de :

(24) Il fait une bronchite por ejemplo, en que uno se refiere a una manifestación particular de la enfermedad.

La categoría /compacto/ permite asimismo la operación atributiva, en la que se realza el valor cualitativo de la noción:

(25) Ça, c'est de la patience!

\section{Conclusión}

En este estudio hemos analizado las formas que actualizan la determinación partitiva como el resultado de una representación mental de la realidad y de la estructura gramatical, o dicho de otra manera, como enunciación enunciada. Si el fenómeno de la enunciación se sitúa en el nivel de la conceptualización teórica, la enunciación enunciada supone dar el paso de la teorización a la praxis inscrita en el enunciado.

Así, se ha visto que la determinación partitiva constituía la marca, inscrita en la enunciación enunciada, de cuatro operaciones enunciativas diferentes:

1) El "prélèvement" cuantitativo/cualitativo sobre una noción

2) La masificación de una noción. Configurada a partir de dos tipos diferentes:

a) la ruptura de la organización interna del referente

b) la homogeneización de las ocurrencias constitutivas del referente

3) La institución de una ocurrencia no nula de la noción

4) La atribución de una noción a otra noción

Estas operaciones y sus respectivas atribuciones a las categorías /denso/, /discreto/, /compacto/26 muestran por último la insuficiencia de la asignación de la determinación partitiva al universo significativo de l' "indénombrable" y la cuantificación.

26. La operación atributiva se encuentra en las tres categorías. 
FUNCIONAMIENTO ENUNCIATIVO DE LA DETERMINACIÓN PARTITIVA EN FRANCÉS

\section{Bibliografia}

\section{A) Gramáticas}

DAMOURETTE, J. et PICHON, E., Des mots à la pensée. Essai de grammaire de la langue française. 1911-1927. (7 vol.) París: D’Artrey, 1987.

BECHADE, H., Syntaxe du français moderne et contemporain. París: PUF, 1986.

BONNARD, H., Code du français courant. París: Magnard, 1981

BRUNOT, F., La pensée et la langue. París: Masson et Cie, 1972 (1922)

CHEVALIER, J.C. et al., Grammaire Larousse du Français Contemporain. París:

Larousse, 1964.

GALICHET, G., Grammaire structurale du français moderne. París: Hatier, 1971.

GOUGENHEIM, G., Système grammatical de la langue française. Ed. D’Artrey, 1969.

GREVISSE, P., Le Bon Usage. París-Gembiloux: Duculot, 1980 (11ème éd.) (1936)

LE BIDOIS, G., et LE BIDOIS, R., (2 vol.), Syntaxe du français moderne. A. \& J. Picard, 1968

MAHMOUDIAN, M., Les modalités nominales. Essai de syntaxe fonctionnelle. París: PUF, 1970.

MARTINET, A., Grammaire fonctionnelle du français. París: Didier, 1979.

SAUVAGEOT, A., Les procédés expressifs du français contemporain. París: Klincksieck, 1957.

TESNIERE, L., Eléments de syntaxe structurale. París: Klincksieck, 1965.

TOGEBY, K., Grammaire française (5 vol.), ed. M. Berg, G. Merad y E. SpangHanssen. Copenhague, 1982-85.

WAGNER, R. L. et PICHON, J., Grammaire du français classique et moderne. París: Hachette, 1962.

WARTBURG, W. von et ZUMTHOR, P., Précis de syntaxe du français contemporain. Berne: Editions Francke, 1958.

B) Teorias y descripciones del sintagma nominal

CULIOLI, A., "A propos de "quelque", S. Fisher et J.J. Franckel, (eds), Linguistique, énonciation. Aspects et détermination. París: Editions de l'Ecole des Hautes Etudes et Sciences Sociales, 1983, pp. 21-29.

GUILLAUME, G., Le problème de l'article et sa solution dans la langue française. París: Hachette, 1919.

DANON-BOILEAU, L. éd., "Détermination, énonciation, référence". Langages 94, 1989.

DAVID, J. et KLEIBER, G. éd., “Déterminants: syntaxe et sémantique”. Actes du Colloque de Metz (6-8 Décembre 1984), París: Klincksieck, 1986. 
FISHER, S. et FRANCKEL, J.J. (eds)., Linguistique, énonciation. Aspects et détermination. París: Editions de l'Ecole des Hautes Etudes en Sciences Sociales, 1983.

GALMICHE, M., "A propos de la distinction massif/comptable", Modèles linguistiques, 1988

GUILLAUME, G., Langage et science du langage. París-Québec: Nizet-P.U. Laval, 1964. Especialmente los dos estudios: "Particularisation et généralisation dans le système des articles français" y "Logique constructive interne du système des articles français".

LÓPEZ ALONSO, C., "Funcionamiento discursivo de la determinación nominal en francés", Comunicación presentada al XIX Simposio de la Sociedad Española de Lingüística, Salamanca 19-21 Diciembre, 1989

NORMAND, C., "Comment l'article cessa d'être un petit mot", Langue Française, 55 (1982) pp. 25-44.

TUTESCU, M., Le groupe nominal et la nominalisation en français moderne. París: Klincksieck, 1972

VATER, H., "Les déterminants: délimitation, syntaxe, sémantique", DRLAV, 25 (1981) pp. 145-173.

WILMET, M., "Les déterminants du nom en français. Essai de synthèse", Langue Française, 57 (1983) pp. 15-33.

WILMET, M., La détermination nominale. Quantification et caractérisation. Presses Universitaires de France, 1986

C) Estudios sobre el partitivo

FREI, H., "Tranches homophones: à propos de l'article partitif", Word, 3 (1960) pp. 317-322.

GALMICHE, M., "Note sur les noms de masse et le partitif", Langue Française, 72 (1986) pp. 40-53.

KUPFERMAN, L., "L'article partitif existe-t-il?", Le Français Moderne, 47 (1979) pp. 1-16.

MARTIN, R., "De la double extensité du partitif", Langue Française 57 (1983) pp. 3442.

MULLER, Cl. "A propos de "de" partitif", Linguisticae Investigationes, I (1977) pp. 167-196.

SPENCE, N. "Partitive and Mass-Nouns in French", Romanische Forschungen, 95 (1983) pp. 1-22.

WILMET, M. "Sur "de" inverseur", Travaux de linguistique et de littérature, XII (1975) pp. 301-323. 